\title{
Hematopoietic cell transplantation (HCT)-specific comorbidity index: a new tool for risk assessment before allogeneic HCT
}

\author{
Mohamed L. Sorror, Michael B. Maris, Rainer Storb, Frederic Baron, Brenda M. Sandmaier, David G. Maloney, and Barry Storer
}

\begin{abstract}
We previously reported that the Charlson Comorbidity Index (CCI) was useful for predicting outcomes in patients undergoing allogeneic hematopoietic cell transplantation (HCT). However, the sample size of patients with scores of 1 or more, captured by the $\mathrm{CCl}$, did not exceed $35 \%$. Further, some comorbidities were rarely found among patients who underwent HCT. Therefore, the current study was designed to (1) better define previously identified comorbidities using pretransplant laboratory data, (2) investigate addi-
\end{abstract}

tional HCT-related comorbidities, and (3) establish comorbidity scores that were suited for HCT. Data were collected from 1055 patients, and then randomly divided into training and validation sets. Weights were assigned to individual comorbidities according to their prognostic significance in Cox proportional hazard models. The new index was then validated. The new index proved to be more sensitive than the $\mathrm{CCl}$ since it captured $62 \%$ of patients with scores more than 0 compared with $12 \%$, respectively. Further, the new index showed better survival prediction than the $\mathrm{CCl}$ (likelihood ratio of $\mathbf{2 3 . 7}$ versus 7.1 and $c$ statistics of 0.661 versus 0.561 , respectively, $P<.001$ ). In conclusion, the new simple index provided valid and reliable scoring of pretransplant comorbidities that predicted nonrelapse mortality and survival. This index will be useful for clinical trials and patient counseling before HCT. (Blood. 2005;106: 2912-2919)

๑ 2005 by The American Society of Hematology

\section{Introduction}

Comorbidity, as defined by Feinstein, ${ }^{1}$ is any distinct additional clinical entity that has existed or may occur during the clinical course of a patient with a primary (index) disease. Comorbidities affect therapeutic plans and posttherapeutic outcomes of the index disease. ${ }^{1}$ In patients with cancer as the index disease, multiple studies have demonstrated the relevance of comorbidities in the prognosis (reviewed in Extermann ${ }^{2}$ ). The number of comorbidities was suggested to increase with aging of cancer patients (reviewed in Extermann ${ }^{3}$ ). In Surveillance, Epidemiology, and End Results (SEER) registry data, $75 \%$ of patients 55 years and older with colon cancer had more than one comorbidity. ${ }^{4}$ However, patients with comorbidities are often excluded from clinical trials, and there are little data on how to translate results from cooperative studies to patients with comorbid diseases. Therefore, the assessment of comorbidities should be integrated into clinical trials, in addition to functional status, particularly since comorbidities and functional status can be used independently to predict outcomes. ${ }^{5-7}$ Efforts to analyze the impact of comorbidities on index diseases are still in their early stages. The interactions between the index diseases and different comorbidities are variable based on the type and degree of organ involvement. Therefore, several indices have introduced a

From the Fred Hutchinson Cancer Research Center, Seattle, WA; and the University of Washington, Seattle, WA.

Submitted May 19, 2005; accepted June 16, 2005. Prepublished online as Blood First Edition Paper, June 30, 2005; DOI 10.1182/blood-2005-05-2004.

Supported in part by grants CA78902, CA92058, CA18029, CA49605, HL36444, and CA15704 from the National Institutes of Health, Department of Health and Human Services (DHHS), Bethesda, MD. M.L.S. was supported by a grant from the Oncology Research Faculty Development Program of the Office of International Affairs of the National Cancer Institute.

M.L.S. designed the study, collected and extracted patients' data, performed the research, analyzed the statistical results, and wrote the paper. M.B.M. provided guidance for the study design, contributed to analysis of the statistical results, and edited the paper. R.S. provided guidance for the study design, has been senior investigator on underlying clinical studies, contributed to analysis way to rate the impact of different comorbidities on the index disease. One of these is the Charlson Comorbidity Index (CCI). The CCI was developed by assigning weights for 19 chronic conditions according to their association with 1-year mortality in a cohort of 559 patients admitted to a general medical center. Then, a summary score based on the sum of the weights was validated in a cohort of breast cancer patients by evaluating the ability of scores to predict mortality. ${ }^{8}$ Later, Charlson added age to her weighted comorbidity scores by assigning an extra point for every decade of age starting at 50 years. ${ }^{9}$ The CCI has been widely used in predicting mortality risks in various medical conditions including solid malignancies. ${ }^{3,10-17}$ We have recently used the CCI in the settings of ablative and nonablative allogeneic hematopoietic cell transplantation (HCT) for patients with hematologic malignancies. In our studies, an adapted form of the CCI successfully predicted the risks of nonrelapse mortality (NRM) in patients receiving hematopoietic cell transplants from unrelated ${ }^{18}$ or related ${ }^{19}$ donors. Those initial observations have been confirmed in several subsequent reports analyzing outcomes after nonmyeloablative HCT. ${ }^{20-23}$ However, some of the comorbidities described by Charlson were rarely encountered in HCT patients due to existing exclusion

of the statistical results, provided oversight, and edited the paper. F.B. contributed to collection of patient data and edited the paper. B.M.S. provided guidance for the study design and edited the paper. D.G.M. provided guidance for the study design and edited the paper. B.S. contributed to the statistical design of the study, performed the statistical analyses, contributed to writing "Patients, materials, and methods," and edited the paper.

An Inside Blood analysis of this article appears at the front of this issue.

Reprints: Rainer Storb, Fred Hutchinson Cancer Research Center, 1100 Fairview Ave N, D1-100, PO Box 19024, Seattle, WA 98109-1024; e-mail: rstorb@fhcrc.org.

The publication costs of this article were defrayed in part by page charge payment. Therefore, and solely to indicate this fact, this article is hereby marked "advertisement" in accordance with 18 U.S.C. section 1734.

(C) 2005 by The American Society of Hematology 
criteria. This was particularly true for hepatic and pulmonary comorbidities. In addition, the CCI did not capture some frequent comorbidities, such as recent infections and psychiatric disturbances. Further, the CCI lacked sensitivity since comorbidities were found in only $35 \%$ of patients, and this percentage was even lower in patients undergoing ablative HCT (12\% in unrelated ${ }^{18}$ and $22 \%$ in related ${ }^{19}$ ablative recipients). Some investigators have pointed out the somewhat limited ability of the CCI to capture comorbidities in patients with primary diagnoses other than hematologic diseases. ${ }^{5,24}$ Others were successful in developing disease-specific comorbidity indices by modifying the original CCI. ${ }^{12,13}$ With the development of nonmyeloablative HCT regimens and improvements in the supportive care after myeloablative HCT regimens, more patients with comorbidities are being offered allogeneic HCT. Therefore, in the current study, we sought to identify those chronic medical conditions, which were important in predicting NRM in $\mathrm{HCT}$ patients, and then integrate these factors into a new scoring system to assess survival probabilities after allogeneic HCT.

\section{Patients, materials, and methods}

The analysis was approved by the institutional review board of the Fred Hutchinson Cancer Research Center (FHCRC, Seattle, WA). Informed consent was provided according to the Declaration of Helsinki.

\section{Patients: data source and patient selection}

This analysis included consecutive and concurrent patients given either nonablative $(\mathrm{n}=294)$ or ablative $(\mathrm{n}=761)$ conditioning followed by hematopoietic cell grafts from related (1997-2003) or unrelated (20002003) donors. Nonablative patients were conditioned with 2 Gy total body irradiation (TBI) either alone $(n=68)$ or preceded by fludarabine $(n=226)$ and were given postgrafting immunosuppression with cyclosporine (CSP) and mycophenolate mofetil (MMF). ${ }^{25-28}$ Ablative patients were conditioned with either busulphan/cyclophosphamide ${ }^{29}$ or cyclophosphamide/TBI ${ }^{30,31}$ and, in almost all cases, were given postgrafting immunosuppression with $\mathrm{CSP} /$ methotrexate. ${ }^{32}$

Diagnoses and clinical grading of acute and chronic graft-versus-host disease (GVHD) were performed using standard criteria. ${ }^{33,34}$ Primary treatment of GVHD consisted of systemic corticosteroids, oral beclomethasone with or without systemic corticosteroids, or reinstitution of CSP.

Early detection of cytomegalovirus antigenemia and preemptive ganciclovir therapy were used for all patients ${ }^{35}$ as were standard prophylaxis against Candida infections (fluconazole), ${ }^{36}$ bacterial infections (ceftazi- dime or ciprofloxacin), Pneumocystis carinii infection (trimethoprimsulfamethoxazole or dapsone), ${ }^{37}$ and herpes simplex virus ${ }^{38}$ and varicella zoster virus ${ }^{39}$ reactivation in serologically positive patients (acyclovir).

Low disease risk included acute leukemia in first complete remission, chronic myeloid leukemia in first chronic phase, myelodysplasia-refractory anemia, and nonmalignant hematologic diseases; while high disease risk included all other diagnoses.

\section{Data collection and modified comorbidities}

Demographic data were obtained from the FHCRC database. Information on comorbidities was extracted from detailed review of the patients' medical charts and laboratory values at the time of HCT. All comorbidities, which were encountered in the 1055 reviewed patients, were included in the current study. Patients were given scores based on the original CCI. ${ }^{8}$ A new HCT-specific comorbidity index (HCT-CI) was then developed as follows: First, definitions of several comorbidities were modified as described in Table 1. Second, comorbidities that were not found in the original CCI were identified as newly investigated or additional comorbidities. Those included bleeding, headache, osteoarthritis, osteoporosis, asthma, obesity, infection, and psychiatric disturbances. The added comorbidities had to be active or requiring treatment at the time of $\mathrm{HCT}$, as defined by Charlson et al. ${ }^{8}$ Third, all other comorbidities were assigned new weights in the HCT-CI using the original definitions in the $\mathrm{CCI},{ }^{8}$ including gastrointestinal disease, coagulopathy, endocrine disease, hypertension, arrhythmia, inflammatory bowel disease, diabetes, cerebrovascular disease, rheumatologic disease, peptic ulcer, and heart valve disease.

\section{Assignment of new comorbidity weights}

In order to develop the new HCT-CI, patients were randomly divided into 2 cohorts. Two thirds of the patients were assigned to a training set to develop the scoring weights $(\mathrm{n}=708)$, and one third was assigned to a validation set $(n=347)$. Integer weights for the HCT-CI were derived from Cox proportional hazards modeling applied to the training set, with NRM over the first 2 years as the outcome. NRM was used instead of survival for development of the scores, since deaths from nonrelapse causes are more likely influenced by pretransplant comorbidities than deaths caused by disease progression or relapse, which were treated as competing risks. Adjusted hazard ratios (HRs) for NRM over the first 2 years after transplantation were calculated for each comorbid condition, controlling for the presence of all coexisting comorbidities as well as for age (younger versus older than 50 years), type of conditioning (nonablative versus ablative), and disease risk (high versus low). The adjusted HRs were converted to integer weights according to the following: comorbidities with adjusted HR of 1.2 or less were dropped from consideration, comorbidities with adjusted HR of 1.3 to 2.0 were assigned a weight of 1 , comorbidities with adjusted HR of 2.1 to 3.0 were assigned a weight of 2 , and

Table 1. Refinement of definitions of comorbidities

\begin{tabular}{|c|c|c|}
\hline Comorbidity & CCI definition & New definition, from $\mathrm{HCT}-\mathrm{Cl}$ \\
\hline Mild pulmonary & Dyspnea on moderate activity & Dyspnea on moderate activity or DLco and/or FEV $181 \%-90 \%$ \\
\hline Moderate pulmonary & Dyspnea on slight activity & Dyspnea on slight activity or DLco and/or FEV $166 \%-80 \%$ \\
\hline Severe pulmonary & Dyspnea at rest or requires oxygen & Dyspnea at rest or requires oxygen or DLco and/or FEV $1 \leq 65 \%$ \\
\hline Cardiac & $\begin{array}{l}\text { Congestive heart failure (symptomatic and requiring } \\
\text { treatment) and myocardial infarction were included } \\
\text { as independent comorbidities, each acquiring a } \\
\text { score of } 1\end{array}$ & $\begin{array}{l}\text { Includes coronary artery disease, }{ }^{*} \text { congestive heart failure, myocardial infarction, or } \\
\text { ejection fraction } \leq 50 \% \text { : one or more acquiring a score of } 1\end{array}$ \\
\hline Mild hepatic & Chronic hepatitis or cirrhosis & Chronic hepatitis, bilirubin $>$ ULN to $1.5 \times$ ULN, or AST/ALT $>$ ULN to $2.5 \times$ ULN \\
\hline Moderate-severe hepatic & Cirrhosis with portal hypertension \pm bleeding varices & Cirrhosis, fibrosis, bilirubin $>1.5 \times$ ULN, or AST/ALT $>2.5 \times$ ULN \\
\hline Mild renal & Serum creatinine $2-3 \mathrm{mg} / \mathrm{dL}$ & Creatinine $1.2-2 \mathrm{mg} / \mathrm{dL}$ \\
\hline Moderate-severe renal & Creatinine $>3 \mathrm{mg} / \mathrm{dL}$, renal dialysis, or renal transplant & Creatinine $>2 \mathrm{mg} / \mathrm{dL}$, renal dialysis, or renal transplant \\
\hline Prior solid tumor & Initially treated in the last $5 \mathrm{y}$ & $\begin{array}{l}\text { Treated at any time point in the patient's past history, excluding nonmelanoma skin } \\
\text { cancer }\end{array}$ \\
\hline
\end{tabular}

To convert creatinine from milligrams per deciliter to micromoles per liter, multiply milligrams per deciliter by 88.4.

${ }^{*}$ One or more vessel-coronary artery stenosis requiring medical treatment, stent, or bypass graft.

DLco indicates diffusion capacity of carbon monoxide; $\mathrm{FEV}_{1}$, forced expiratory volume in one second; ULN, upper limit of normal; AST, aspartate aminotransferase; and ALT, alanine aminotransferase. 
comorbidities with adjusted HR of 3.1 or more were assigned a weight of 3 . The HCT-CI score was the sum of these integer weights. The HCT-CI scores were further collapsed into 3 risk groups: 0 (low risk), 1 to 2 (intermediate risk), and 3 or more (high risk). For comparative purposes, the original CCI was also reduced to 3 risk groups: 0,1 , and 2 or more.

\section{Model validation}

The scores of the new HCT-CI were applied to patients of the validation set to test their ability to predict for HR and cumulative incidence of NRM. The new HCT-CI was then compared with the original CCI for prediction of both NRM and survival. Survival was added as an end point for comparison between the 2 indices since the CCI scores were derived from Cox proportional hazards modeling for survival. ${ }^{8}$ Cumulative incidence ${ }^{40}$ curves for NRM and Kaplan-Meier curves for survival were computed for each risk group defined by the 2 indices. Likelihood ratio statistics from proportional hazards models were computed for both indices for events over the first 2 years. Although a larger likelihood ratio statistic indicated a better fit to the data, the likelihood ratio by itself had no intuitive interpretation in terms of prediction. For this purpose, we also computed the $c$-statistic. ${ }^{41}$ For a continuous predictor, the $c$-statistic could be interpreted as the probability that a random pair of observations whose event times could be ordered would have a concordant ordering of the predictor. A value of 1.0 indicated perfect predictive discrimination, whereas a value of 0.5 indicated no ability to discriminate. If the data were collapsed to indicate a binary outcome (survival larger or smaller than a fixed point in time), then the $c$-statistic was also interpretable as the area under a receiver operating characteristic curve. Pairs of observations whose ordering could not be assigned because of censoring were excluded from the calculation. The $c$-statistic was computed for NRM and survival, based on both time to event over the first 2 years and as a binary outcome indicating event times before/after 1 year or 2 years. For computing $c$-statistic for NRM, patients were censored at the time of relapse/progression. Standard errors for the $c$-statistic were estimated by applying a bootstrap procedure to the validation dataset, using 100 bootstrap samples. Similarly, the standard error for the difference in $c$-statistic between the CCI and HCT-CI was estimated from the bootstrap samples and used to calculate a $\mathrm{z}$-score and $P$ value for the difference.

\section{Results}

\section{Patient characteristics}

Characteristics of the 1055 patients included in this study are shown in Table 2. A majority of patients received cyclophosphamide/ busulphan conditioning (43\%), 29\% received cyclophosphamide/ TBI (12-14.4 Gy), 22\% received fludarabine/TBI (2 Gy), and 6\% received 2 Gy TBI. GVHD prophylaxis was MTX/CSP in $69 \%$ of patients and MMF/CSP in $31 \%$. Myeloid malignancies constituted the majority of diagnoses (66\%). Forty-one percent of the patients had high risk disease. Median age was 44.8 years. Related grafts were used in $58 \%$ of patients, while the remainder received unrelated grafts. Thirteen percent of patients had preceding myeloablative HCT, of which $2 \%$ were allogeneic, $6 \%$ were failed autologous, and 5\% were planned autologous. A majority of patients received peripheral blood stem cell grafts (71\%).

\section{The HCT-CI}

The prevalence of various comorbidities together with the cumulative incidence and multivariate HR for 2-year NRM, as predicted by each comorbidity, are shown in Table 3 . The definitions of comorbidities included in the HCT-CI, along with the integer weights compared with those of the CCI, are shown in Table 4. Pulmonary and hepatic abnormalities, as defined by laboratory data, were the most frequent comorbidities in the new HCT-CI and,
Table 2. Patient and disease characteristics

\begin{tabular}{|c|c|}
\hline Characteristic & Data \\
\hline \multicolumn{2}{|l|}{ Conditioning regimens, $\%$} \\
\hline 2 Gy TBI & 6 \\
\hline Fludarabine + 2 Gy TBI & 22 \\
\hline $\mathrm{CY}+$ at least $12 \mathrm{~Gy}$ TBI & 29 \\
\hline $\mathrm{BU}+\mathrm{CY}$ & 43 \\
\hline \multicolumn{2}{|c|}{ Postgrafting immunosuppression, \% } \\
\hline $\mathrm{CSP}+\mathrm{MMF}$ & 18 \\
\hline $\mathrm{CSP}+\mathrm{MTX}$ & 82 \\
\hline \multicolumn{2}{|l|}{ Donor type, \% } \\
\hline Related donor & 58 \\
\hline Unrelated donor & 42 \\
\hline \multicolumn{2}{|l|}{ Diagnoses, \% } \\
\hline AML & 27 \\
\hline CML & 20 \\
\hline MDS & 19 \\
\hline ALL & 10 \\
\hline NHL & 9 \\
\hline MM & 6 \\
\hline CLL & 4 \\
\hline HD & 2 \\
\hline Nonmalignant hematologic* & 3 \\
\hline \multicolumn{2}{|l|}{ Disease risk group, \%† } \\
\hline High & 41 \\
\hline Low & 59 \\
\hline \multicolumn{2}{|l|}{ Age at transplantation } \\
\hline Median (range), y & $44.8(0.8-72.7)$ \\
\hline \multicolumn{2}{|c|}{ Preceding myeloablative HCT, \% } \\
\hline Allogeneic & 2 \\
\hline Failed autologous & 6 \\
\hline Planned autologous & 5 \\
\hline \multicolumn{2}{|l|}{ Hematopoietic cell source, \% } \\
\hline G-PBMC & 71 \\
\hline Marrow & 29 \\
\hline \multicolumn{2}{|l|}{ Male/female, \% } \\
\hline Patients & $56 / 44$ \\
\hline Donors & $51 / 49$ \\
\hline
\end{tabular}

$\mathrm{n}=1055$.

TBI indicates total body irradiation; CY, cyclophosphamide; BU, busulfan; CSP, cyclosporine; MMF, mycophenolate mofetil; MTX, methotrexate; AML, acute myeloid leukemia; CML, chronic myeloid leukemia; MDS, myelodysplastic syndromes; ALL, acute lymphoblastic leukemia; NHL, non-Hodgkin lymphoma; MM, multiple myeloma; CLL, chronic lymphocytic leukemia; HD, Hodgkin disease; HCT, hematopoietic cell transplantation; and G-PBMC, granulocyte colony stimulating factor-mobilized peripheral blood mononuclear cells.

*Immunodeficiency syndrome, chronic granulomatous disease, congenital dyserythropoietic anemia, paroxysmal nocturnal hemoglobinuria, polycythemia vera, thalassemic syndrome, and sickle cell anemia.

tLow indicates acute leukemia in first remission, CML in first chronic phase, MDS-refractory anemia, or nonmalignant hematologic disease; while high indicates all other diagnoses.

together with prior solid tumor and heart valve disease, had the highest assigned weights. By comparing conditions in the newly derived HCT-CI with those of the CCI, a number of comorbidities increased in relative importance, including arrhythmia, inflammatory bowel disease, rheumatologic disease, peptic ulcer, moderate and severe pulmonary comorbidity, prior solid tumor, and heart valve disease. Other conditions, including cardiac disease, diabetes mellitus, cerebrovascular disease, mild hepatic comorbidity, moderate/severe renal comorbidity, and moderate/severe hepatic comorbidity, had comparable weights both in the HCT-CI and the original CCI. Of the 8 comorbidities tested that were not specified by Charlson, obesity, peritransplant infections, and psychiatric disturbances were assigned weights in the HCT-CI, whereas bleeding, headache, osteoarthritis, osteoporosis, and asthma were not because of low predictive HR for NRM $(0.0,0.3,0.4,0.7$, and 1.1, 
Table 3. Prevalence of comorbidities among 708 patients included in the training set with cumulative incidences and multivariate HRs of comorbidities for 2-year NRM

\begin{tabular}{|c|c|c|c|}
\hline Comorbidity & Prevalence, $\%$ & 2-year NRM, \% & Multivariate HR $^{\star}$ \\
\hline Bleeding $†$ & 1 & 0 & 0.0 \\
\hline Headache† & 4 & 8 & 0.3 \\
\hline Osteoarthritis $†$ & 1 & 11 & 0.4 \\
\hline Gastrointestinal disease & 9 & 20 & 0.5 \\
\hline Coagulopathy & 6 & 30 & 0.6 \\
\hline Osteoporosis† & 1 & 75 & 0.7 \\
\hline Renal, mild $\ddagger$ & 10 & 24 & 0.8 \\
\hline Endocrine disease & 5 & 31 & 1.0 \\
\hline Asthma† & 2 & 14 & 1.1 \\
\hline Pulmonary, mild $\ddagger$ & 16 & 18 & 1.1 \\
\hline Hypertension & 10 & 33 & 1.2 \\
\hline Arrhythmia & 5 & 35 & 1.3 \\
\hline Cardiac $\ddagger$ & 5 & 37 & 1.3 \\
\hline Inflammatory bowel disease & 1 & 31 & 1.3 \\
\hline Diabetes & 3 & 50 & 1.6 \\
\hline Cerebrovascular disease & $<1$ & 67 & 1.6 \\
\hline Psychiatric disturbance $†$ & 9 & 29 & 1.8 \\
\hline Hepatic, mild $\ddagger$ & 16 & 30 & 1.9 \\
\hline Obesity† & 2 & 33 & 1.9 \\
\hline Infection† & 4 & 40 & 1.9 \\
\hline Rheumatologic & 4 & 45 & 2.3 \\
\hline Peptic ulcer & 1 & 38 & 2.5 \\
\hline Renal, moderate/severeł & 2 & 42 & 2.6 \\
\hline Pulmonary, moderateł & 24 & 36 & 3.0 \\
\hline Prior solid tumorł & 2 & 38 & 3.1 \\
\hline Heart valve disease & 2 & 33 & 3.3 \\
\hline Pulmonary, severe & 9 & 39 & 3.7 \\
\hline Hepatic, moderate/severeł & 4 & 43 & 3.9 \\
\hline Peripheral vascular disease & 0 & - & - \\
\hline
\end{tabular}

\section{— indicates not applicable.} type.

*Adjusted for all other comorbidities, age, high disease risk, and conditioning

†Newly investigated comorbidities.

$\neq$ Comorbidities with modified definitions compared with the original $\mathrm{CCl}$.

respectively). For peripheral vascular disease, no conclusions could be drawn since none of the current patients had this comorbidity at the time of HCT.

The HCT-CI scores ranged from 0 to 11 in the training set, compared with 0 to 4 for the original CCI scores.

\section{Validation of the new HCT-Cl}

The newly developed HCT-CI scores were then calculated for patients in the independent validation set $(n=347)$. The HR and 2-year cumulative incidence of NRM are summarized by HCT-CI scores for the training and validation sets in Table 5. Due to small numbers, patients with scores of 4 or more were collapsed into one group. Probably due to the smaller number of patients, there was an apparent shrinkage of the discriminatory ability for NRM among various risk groups between the training and validation sets; nevertheless, the HCT-CI continued to be strongly associated with NRM.

\section{Comparing the new HCT-Cl with the original $\mathrm{CCl}$}

For the purpose of comparison, the 2 comorbidity indices were each separated into 3 risk groups. For the HCT-CI these were low $(0)$, intermediate (1-2), and high ( $\geq 3$ ) and for the CCI, low (0), intermediate (1), and high $(\geq 2)$. Results for NRM and survival are summarized by risk group in Table 6 and Figure 1. It was clear that the risk groups based on the HCT-CI scores were more evenly distributed and provided better discrimination of NRM risks than the risk groups defined by the CCI. Although the risk groups for both indices provided some discrimination for survival, the CCI had a lower overall predictive value for survival because of the concentration of nearly $90 \%$ of scores in the 0 score category.

Statistical comparisons between the HCT-CI and CCI are summarized in Table 7. There was stronger association of higher HCT-CI scores with worse NRM and survival based on the likelihood ratio. In addition, the $c$-statistics were significantly higher using the HCT-CI compared with the CCI, whether as an overall measure of association (the percent of pairs of patients where the patient with the higher comorbidity score failed first) or for association with a dichotomized end point at 1 year or 2 years (the percent of pairs of patients where the patient failing before 1 year or 2 years had a higher comorbidity score than the patient failing after that time).

\section{Correlation between causes of death and most common comorbidities}

Pulmonary comorbidities, as defined by impairments of forced expiratory volume in one second $\left(\mathrm{FEV}_{1}\right)$ and/or diffusion capacity of carbon monoxide (DLco), and hepatic comorbidities, as defined by elevations of hepatic function tests, were the most prevalent comorbidities captured by the new HCT-CI. There were 347 patients with moderate (score 2) or severe (score 3) pulmonary comorbidities, and 122 died from nonrelapse causes. Causes of NRM among these 122 patients included pulmonary toxicities (24\%), GVHD (11\%), and infections (45\%) with (18\%) or without (27\%) GVHD (infections involved the lungs in almost half of the cases), and $20 \%$ died from other causes.

Mild (score 1) and moderate-severe (score 3 ) hepatic comorbidities were encountered in 215 patients, of whom 72 died of nonrelapse causes. Twenty-one percent of these 72 patients died from GVHD; $10 \%$, from hepatic causes; $36 \%$, from infections with or without GVHD; $15 \%$, from pulmonary causes; and $18 \%$, from other causes.

Overall, fewer nonablative than ablative patients had scores of 0 ( $29 \%$ versus $41 \%$ ) and 1 to 2 (31\% versus $35 \%$ ), while more nonablative than ablative patients had scores of 3 or more ( $40 \%$ versus $24 \%$ ).

\section{Discussion}

We previously reported on the importance of pretransplant comorbidities for outcomes after allogeneic $\mathrm{HCT}^{18,19}$ using the CCI, given that it was a validated and simple scoring system for comorbidities. The studies were confined both by small sample sizes and the limited ability of the CCI to comprehensively capture comorbidities, particularly in patients given ablative conditioning. The current study refined comorbidity definitions, added newly identified comorbidities, and evaluated each comorbidity category by Cox regression hazard models. A new index of scores for each comorbidity was then developed and validated in another randomly selected group of HCT recipients. We found that the HCT-CI captured more pretransplant comorbidities and provided better assessment of NRM and survival risks compared with the original CCI.

The adverse impact of comorbidities on cancer patients was likely due to the physiologic burden of chronic disease and its interactions with cancer and cancer treatment. ${ }^{42}$ Therefore, increased severity of comorbidities increased the risk of toxicities in response to specific treatments and, hence, shortened life expectancy and canceled gains derived from specific therapies. ${ }^{43}$ There has been ample evidence in the literature that comorbidities and 
Table 4. Definitions of comorbidities included in the HCT-Cl and HCT-Cl scores compared with original CCI scores

\begin{tabular}{|c|c|c|c|}
\hline Comorbidity & Definitions of comorbidities included in the new $\mathrm{HCT}-\mathrm{Cl}$ & HCT-Cl weighted scores & Original $\mathrm{CCl}$ scores* \\
\hline Arrhythmia & Atrial fibrillation or flutter, sick sinus syndrome, or ventricular arrhythmias & 1 & 0 \\
\hline Cardiac $\ddagger$ & $\begin{array}{l}\text { Coronary artery disease, } \S \text { congestive heart failure, myocardial infarction, } \\
\text { or } \mathrm{EF} \leq 50 \%\end{array}$ & 1 & 1 \\
\hline Inflammatory bowel disease & Crohn disease or ulcerative colitis & 1 & 0 \\
\hline Diabetes & Requiring treatment with insulin or oral hypoglycemics but not diet alone & 1 & 1 \\
\hline Cerebrovascular disease & Transient ischemic attack or cerebrovascular accident & 1 & 1 \\
\hline Psychiatric disturbance & Depression or anxiety requiring psychiatric consult or treatment & 1 & Not included \\
\hline Hepatic, mild $\ddagger$ & $\begin{array}{l}\text { Chronic hepatitis, bilirubin }>\text { ULN to } 1.5 \times \text { ULN, or AST/ALT > ULN to } \\
2.5 \times \text { ULN }\end{array}$ & 1 & 1 \\
\hline Obesity† & Patients with a body mass index $>35 \mathrm{~kg} / \mathrm{m}^{2}$ & 1 & Not included \\
\hline Infection† & Requiring continuation of antimicrobial treatment after day 0 & 1 & Not included \\
\hline Rheumatologic & SLE, RA, polymyositis, mixed CTD, or polymyalgia rheumatica & 2 & 1 \\
\hline Peptic ulcer & Requiring treatment & 2 & 1 \\
\hline Moderate/severe renalł & Serum creatinine $>2 \mathrm{mg} / \mathrm{dL}$, on dialysis, or prior renal transplantation & 2 & 2 \\
\hline Moderate pulmonary $\ddagger$ & DLco and/or FEV $166 \%-80 \%$ or dyspnea on slight activity & 2 & 1 \\
\hline Prior solid tumor & $\begin{array}{l}\text { Treated at any time point in the patient's past history, excluding } \\
\text { nonmelanoma skin cancer }\end{array}$ & 3 & 2 \\
\hline Heart valve disease & Except mitral valve prolapse & 3 & 0 \\
\hline Severe pulmonary $\ddagger$ & DLco and/or FEV $1 \leq 65 \%$ or dyspnea at rest or requiring oxygen & 3 & 1 \\
\hline Moderate/severe hepatic $\ddagger$ & Liver cirrhosis, bilirubin $>1.5 \times$ ULN, or AST/ALT $>2.5 \times$ ULN & 3 & 3 \\
\hline
\end{tabular}

To convert creatinine from milligrams per deciliter to micromoles per liter, multiply milligrams per deciliter by 88.4.

EF indicates ejection fraction; ULN, upper limit of normal; SLE, systemic lupus erythmatosis; RA, rheumatoid arthritis; CTD, connective tissue disease; DLco, diffusion capacity of carbon monoxide.

${ }^{*}$ Definitions of comorbidities included in the original $\mathrm{CCl}$ are defined in the appendix of a prior publication. ${ }^{8}$

†Newly investigated comorbidities.

†Comorbidities with modified definitions compared with the original CCI.

$\S$ One or more vessel-coronary artery stenosis requiring medical treatment, stent, or bypass graft.

performance scales both independently predicted for outcome. ${ }^{5}$ However, contrary to functional status, comorbidities presented the unique challenge of being a multidimensional variable, since diseases influencing mortality might not be the same as diseases influencing function or tolerance to treatment. ${ }^{2}$ Translating comorbidities into weighted scores appeared to be the best way to use comorbidities for prediction of outcome. ${ }^{2,8,44,45}$

In the current study, we sought to modify the $\mathrm{CCI}$ in a way to improve sensitivity and specificity for predicting risks of NRM after HCT. The primary modification was refinement of several comorbidity definitions, which was done by introducing objective laboratory and functional testing data, thereby incorporating subclinical organ impairments that could result in partial compromise in patients subjected to the intensive physiologic challenge of HCT. Pulmonary function tests were used to define different grades of pulmonary comorbidities. Similarly, liver function tests and cardiac ejection fraction were added to definitions of hepatic and cardiac comorbidities. This introduction of laboratory data constituted the main change for increasing the sensitivity of the new HCT-CI, in particular since pulmonary and hepatic comorbidities achieved the highest prevalence among our transplant populations. The new
HCT-CI captured $62 \%$ of patients with scores more than 0 compared with $12 \%$ captured by the original CCI. In particular, $59 \%$ of ablative patients had scores more than 0 using the HCT-CI compared with $10 \%$ by the original CCI.

The improvement in the new HCT-CI over the original CCI was demonstrated by likelihood ratio and $c$ statistics. The improvement was likely related to the assignment of comorbidity weights specific to patients who underwent transplantation. Several common comorbidities were assigned higher weights than in the original CCI, likely reflecting their importance for tolerating HCT. In addition, new comorbidities, such as infections, obesity, and psychiatric disturbances, were found to be clinically relevant. The use of laboratory data in the new HCT-CI accounted for larger numbers of patients with scored comorbidities, which added to the utility of the new HCT-CI over the original CCI.

In all cases, the $c$-statistic calculated for the HCT-CI was 7 to 15 percentage points higher than that for the CCI, with the value for the CCI being only slightly better than a coin flip ( 0.50$)$. Although the difference between HCT-CI and CCI was highly statistically significant, it should be noted that the magnitude of the $c$-statistic for the HCT-CI reflected a fairly modest level of prediction. This was not

Table 5. The new HCT-Cl scores and prediction for NRM and survival the training versus the validation set

\begin{tabular}{|c|c|c|c|c|c|c|}
\hline \multirow[b]{3}{*}{ Score } & \multicolumn{3}{|c|}{ Training set } & \multicolumn{3}{|c|}{ Validation set } \\
\hline & \multirow[b]{2}{*}{ Patients, \% } & \multicolumn{2}{|c|}{ NRM } & \multirow[b]{2}{*}{ Patients, \% } & \multicolumn{2}{|c|}{ NRM } \\
\hline & & $\mathrm{HR}^{*}(95 \% \mathrm{Cl})$ & 2-year, \% & & $\mathrm{HR}^{\star}(95 \% \mathrm{Cl})$ & 2-year, \% \\
\hline 0 & 38 & 1 & 9 & 38 & 1 & 14 \\
\hline 1 & 17 & $1.66(0.9-3.1)$ & 14 & 18 & $1.57(0.7-3.3)$ & 22 \\
\hline 2 & 17 & $3.48(2.0-6.0)$ & 27 & 17 & $1.26(0.6-2.8)$ & 19 \\
\hline 3 & 17 & $6.09(3.7-10.1)$ & 41 & 15 & $3.95(2.1-7.5)$ & 41 \\
\hline 4 or more & 11 & $6.93(4.0-12.0)$ & 43 & 13 & $3.05(1.5-6 . .2)$ & 40 \\
\hline
\end{tabular}

For the training set, $\mathrm{n}=708$; for the validation set, $\mathrm{n}=346$.

${ }^{*}$ Adjusted for age, disease risk, and conditioning. 
Table 6. HCT-Cl versus $\mathrm{CCl}$ for prediction of 2-year NRM and survival in the validation set

\begin{tabular}{|c|c|c|c|c|c|c|c|c|c|c|c|}
\hline \multirow[b]{3}{*}{ Score } & \multicolumn{5}{|c|}{$\mathrm{HCT}-\mathrm{Cl}$} & \multirow[b]{3}{*}{ Score } & \multirow[b]{3}{*}{ No. } & \multicolumn{4}{|c|}{$\mathrm{CCl}$} \\
\hline & \multirow[b]{2}{*}{ No. } & \multicolumn{2}{|c|}{ NRM } & \multicolumn{2}{|c|}{ Survival } & & & \multicolumn{2}{|c|}{ NRM } & \multicolumn{2}{|c|}{ Survival } \\
\hline & & $\mathrm{HR}^{\star}(95 \% \mathrm{Cl})$ & $\begin{array}{c}\text { 2-year, } \\
\%\end{array}$ & $\mathrm{HR}^{\star}(95 \% \mathrm{Cl})$ & $\begin{array}{c}\text { 2-year, } \\
\%\end{array}$ & & & $\mathrm{HR}^{\star}(95 \% \mathrm{Cl})$ & $\begin{array}{c}\text { 2-year, } \\
\%\end{array}$ & $\mathrm{HR}^{*}(95 \% \mathrm{Cl})$ & $\begin{array}{c}\text { 2-year, } \\
\%\end{array}$ \\
\hline 0 & 38 & 1.0 & 14 & 1.0 & 71 & 0 & 87 & 1.0 & 23 & 1.0 & 59 \\
\hline 1 to 2 & 34 & $1.42(0.8-2.7)$ & 21 & $1.31(0.8-2.0)$ & 60 & 1 & 10 & $1.25(0.6-2.5)$ & 29 & $1.32(0.8-2.2)$ & 49 \\
\hline 3 or more & 28 & $3.54(2.0-6.3)$ & 41 & $2.69(1.8-4.1)$ & 34 & $\geq 2$ & 3 & $1.46(0.5-4.7)$ & 25 & $2.78(1.4-5.6)$ & 17 \\
\hline
\end{tabular}

${ }^{*}$ Adjusted for age, disease risk, and conditioning.

surprising given the multiple factors that also contributed to both NRM and survival and the smaller patient sample in the validation set.

Even though the HCT-CI scores were highly effective in predicting outcome of patients after HCT, other major pretransplant factors played significant roles. Age and disease stage of a specific hematologic malignancy were additional factors that should be considered when determining HCT risks. Age has been used independently to determine if patients should be referred for HCT and to stratify patients for the different intensity conditioning regimens. ${ }^{46-49}$ For that reason and also since age is a demographic variable, we decided not to include age as a scored comorbidity in the HCT-CI. Instead, in the current study, age was used to adjust the Cox regression hazards' modeling for developing the new HCT-CI.

We and other investigators had shown the importance of single organ comorbidities and abnormal laboratory data, as markers of organ dysfunction, on outcome of patients who underwent transplantation. ${ }^{50-52}$ However, none have attempted to evaluate multiple comorbidities into a scoring system with predictive power to determine survival risks after HCT. On the other hand, some disease-specific prognostic scoring systems ${ }^{53,54}$ have been developed for assessing patient risk prior to specific therapies including HCT. However, none of those scores took into account the impact of different comorbidities on outcome.

There were several limitations to our study. The first was the retrospective nature of the data collection. Since we relied on data recorded in medical charts, some potentially important data might not have been recorded and hence not been included in this analysis. However, the introduction of laboratory and functional
A
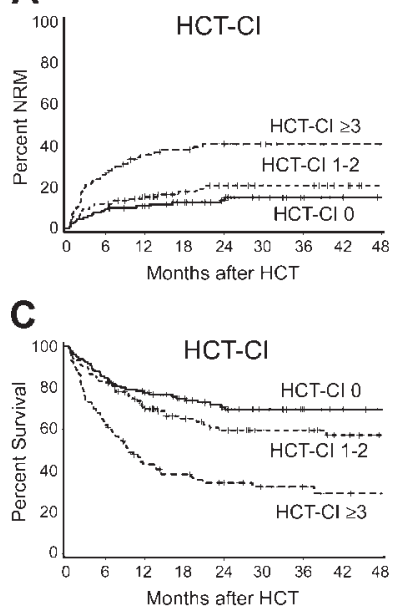

B

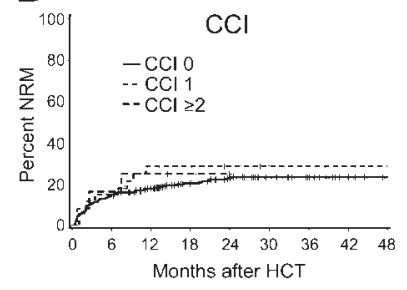

D

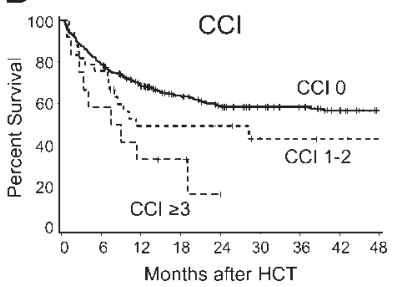

Figure 1. The HCT-Cl compared with the CCl. Cumulative incidences of nonrelapse mortality (NRM) as stratified by the (A) new HCT-Cl compared with (B) the original $\mathrm{CCl}$, and Kaplan-Meier estimates of survival as stratified by (C) the new $\mathrm{HCT}-\mathrm{Cl}$ compared with (D) the original $\mathrm{CCl}$ among patients of the validation set. Only $13 \%$ of patients had scores of 1 or more when scored by the original $\mathrm{CCl}$ compared with $62 \%$ when scored by the new $\mathrm{HCT}-\mathrm{Cl}$. data, most of which were stored in the database, has likely reduced the possibility of missing comorbidities. To better address this problem, new protocols include prospective scoring of enrolled patients. The second limitation was that patients from the same institution were used to validate the new HCT-CI. Efforts are under way to further validate the HCT-CI on patients who underwent transplantation in other institutions. The third limitation was the lack of interrater and test-retest reliabilities in this study. However, reports on the original CCI had previously shown both good interrater reliability between 0.74 and 0.945 by interclass correlation coefficient in older cancer patients ${ }^{5,55}$ and good test-retest reliability of $0.92 .{ }^{56}$ Since we used many of the definitions of the CCI with additional refinements based on laboratory and functional testing, which were not subject to investigator variations, it was likely that the HCT-CI had at least the same interrater reliability as the original CCI. We plan to test the HCT-CI reliability among different investigators located in different institutions.

The current HCT-CI could be applied to a number of settings. Our study identified prevalent and prognostically important coexisting illnesses that should be measured and considered in clinical trials and HCT registries. This would help to extrapolate the results of transplant recipients with significant comorbidities to the entire spectrum of HCT patients. A second application of this HCT-CI involves the consideration of medical evidence for individual patients at the bedside. The index can be widely used by referring physicians, oncologists, and hematologists when assessing the risks of patients with hematologic diseases for specific therapies and particularly referral for HCT. Currently, we investigate the importance of the new HCT-CI on outcomes in disease-specific patients given HCT. The third application includes a wide area of research concerning the correlation between comorbidities captured by the HCT-CI and different complications after HCT, such as infections, GVHD, and respiratory or hepatic

Table 7. Comparisons between the $\mathrm{HCT}-\mathrm{Cl}$ and the $\mathrm{CCl}$ in the validation set $(n=347)$ for events within first 2 years

\begin{tabular}{lccc}
\hline & $\begin{array}{c}\text { HCT-Cl for } \\
\text { 3 risk groups }\end{array}$ & $\begin{array}{c}\text { CCl for } \\
\text { 3 risk groups }\end{array}$ & $\boldsymbol{P}^{*}$ \\
\hline Nonrelapse mortality & & & \\
c-statistic (SE*), overall & $0.649(0.029)$ & $0.520(0.019)$ & $<.001$ \\
c-statistic (SE*), yes/no 2 y & $0.685(0.037)$ & $0.532(0.023)$ & $<.001$ \\
c-statistic (SE*), yes/no 1 y & $0.692(0.036)$ & $0.546(0.025)$ & $<.001$ \\
Likelihood ratio & 26.6 & 1.2 & \\
Adjusted likelihood ratio† & 21.4 & 0.7 & \\
Overall survival & & & $<.001$ \\
c-statistic (SE*), overall & $0.624(0.021)$ & $0.536(0.015)$ & \\
c-statistic (SE*), yes/no 2 y & $0.657(0.031)$ & $0.548(0.019)$ & $<.001$ \\
c-statistic (SE*), yes/no 1 y & $0.661(0.030)$ & $0.561(0.021)$ & \\
Likelihood ratio & 31.8 & 7.6 & \\
Adjusted likelihood ratio† & 23.7 & 7.1 & \\
\hline
\end{tabular}

*Estimated from 100 bootstrap samples

†Adjusted for age, disease risk, and conditioning. 
failure. The final application will be stratifying patients for eligibility of nonablative versus ablative HCT.

In summary, we have developed a new tool for capturing pretransplant comorbidities that could be used in predicting outcomes and stratifying patients for HCT. In the future, we will further validate this index on patients from different institutions and compare its performance to comorbidity indices other than the CCI, such as The Cumulative Illness Rating Scale and the Adult Comorbidity Evaluation (ACE-27). Further, it would be of interest to develop a scaling system for increments of age and different stages of diseases to be used in concert with the HCT-CI in assessing pretransplant patient risk for failure of disease-free survival.

\section{Acknowledgments}

The authors wish to thank Dr Karen Syrjala, associate professor for Psychiatry and Behavioral Sciences at University of Washington and FHCRC, for her valuable advice. We also thank the data coordinators, Chris Davis, Heather Hildebrant, and Deborah Bassuk, and the study nurses, Steve Minor, Mary Hinds, and John Sedgwick, for their invaluable help in making the study possible. Bonnie Larson, Helen Crawford, and Sue Carbonneau provided invaluable help with article preparation. We also wish to thank all the Transplant Teams.

\section{References}

1. Feinstein AR. The pre-therapeutic classification of co-morbidity in chronic disease. J Chron Dis. 1970;23:455-468.

2. Extermann M. Measurement and impact of comorbidity in older cancer patients. Crit Rev Oncol Hematol. 2000;35:181-200.

3. Extermann M. Measuring comorbidity in older cancer patients (review). Eur J Cancer. 2000;36: 453-471.

4. Yancik R, Wesley MN, Ries LAG, et al. Comorbidity and age as predictors of risk for early mortality of male and female colon carcinoma patients. Cancer. 1998;82:2123-2134.

5. Extermann M, Overcash J, Lyman GH, Parr J, Balducci L. Comorbidity and functional status are independent in older cancer patients. J Clin Oncol. 1998;16:1582-1587.

6. Finkelstein DM, Ettinger DS, Ruckdeschel JC. Long-term survivors in metastatic non-small-cell lung cancer: an Eastern Cooperative Oncology Group Study. J Clin Oncol. 1986;4:702-709.

7. Keller BK, Potter JF. Predictors of mortality in outpatient geriatric evaluation and management clinic patients. J Gerontol. 1994;49:M246-M251.

8. Charlson ME, Pompei P, Ales KL, MacKenzie CR A new method of classifying prognostic comorbidity in longitudinal studies: development and validation. J Chronic Dis. 1987;40:373-383.

9. Charlson M, Szatrowski TP, Peterson J, Gold J. Validation of a combined comorbidity index. J Clin Epidemiol. 1994;47:1245-1251.

10. Di lorio B, Cillo N, Cirillo M, De Santo NG. Charlson Comorbidity Index is a predictor of outcomes in incident hemodialysis patients and correlates with phase angle and hospitalization. Int J Artif Organs. 2004;27:330-336.

11. Goldstein LB, Samsa GP, Matchar DB, Horner RD. Charlson Index comorbidity adjustment for ischemic stroke outcome studies. Stroke. 2004; 35:1941-1945.

12. Hemmelgarn BR, Manns BJ, Quan H, Ghali WA Adapting the Charlson Comorbidity Index for use in patients with ESRD. Amer J Kidney Dis. 2003; 42:125-132.

13. Sachdev M, Sun JL, Tsiatis AA, Nelson CL, Mark $\mathrm{DB}$, Jollis JG. The prognostic importance of comorbidity for mortality in patients with stable coronary artery disease. J Am Coll Cardiol. 2004;43: 576-582.

14. Lubke T, Monig SP, Schneider PM, Holscher AH, Bollschweiler E. Does Charlson-comorbidity index correlate with short-term outcome in patients with gastric cancer? [German]. Zentralblatt für Chirurgie. 2003;128:970-976.

15. Firat S, Byhardt RW, Gore E. Comorbidity and Karnofksy performance score are independent prognostic factors in stage III non-small-cell lung cancer: an institutional analysis of patients treated on four RTOG studies: Radiation Therapy Oncology Group. Int J Radiat Oncol Biol Phys. 2002;54:357-364.
16. Sabin SL, Rosenfeld RM, Sundaram K, Har-el G, Lucente FE. The impact of comorbidity and age on survival with laryngeal cancer. Ear Nose Throat J. 1999;78:578, 581-584.

17. Singh B, Bhaya M, Stern J, et al. Validation of the Charlson comorbidity index in patients with head and neck cancer: a multi-institutional study. Laryngoscope. 1997;107:1469-1475.

18. Sorror ML, Maris MB, Storer B, et al. Comparing morbidity and mortality of HLA-matched unrelated donor hematopoietic cell transplantation after nonmyeloablative and myeloablative conditioning: influence of pretransplant comorbidities. Blood. 2004;104:961-968.

19. Diaconescu R, Flowers CR, Storer B, et al. Morbidity and mortality with nonmyeloablative compared to myeloablative conditioning before hematopoietic cell transplantation from HLA matched related donors. Blood. 2004;104:1550-1558.

20. Baron F, Maris MB, Sandmaier BM, et al. Graftversus-tumor effects after allogeneic hematopoietic cell transplantation with nonmyeloablative conditioning. J Clin Oncol. 2005;23:1993-2003.

21. Maris MB, Sandmaier BM, Storer BE, et al. Allogeneic hematopoietic cell transplantation after fludarabine and 2 Gy total body irradiation for relapsed and refractory mantle cell lymphoma. Blood. 2004;104:3535-3542.

22. Mielcarek M, Burroughs L, Leisenring W, et al. Prognostic relevance of "early-onset" graftversus-host disease following nonmyeloablative hematopoietic cell transplantation. Br J Haematol. 2005;129:381-391.

23. Sorror ML, Maris MB, Sandmaier BM, et al. Hematopoietic cell transplantation after nonmyeloablative conditioning for advanced chronic lymphocytic leukemia. J Clin Oncol. Published ahead of print on April 4, 2005, as 10.1200/JCO.2005.04.569-

24. Rochon PA, Katz JN, Morrow LA, et al. Comorbid illness is associated with survival and length of hospital stay in patients with chronic disability: a prospective comparison of three comorbidity indices. Med Care. 1996;34:1093-1101.

25. Feinstein LC, Sandmaier BM, Hegenbart U, et al. Non-myeloablative allografting from human leucocyte antigen-identical sibling donors for treatment of acute myeloid leukaemia in first complete remission. Br J Haematol. 2003;120:281-288.

26. Maloney DG, Molina AJ, Sahebi F, et al. Allografting with nonmyeloablative conditioning following cytoreductive autografts for the treatment of patients with multiple myeloma. Blood. 2003;102: 3447-3454.

27. Maris MB, Niederwieser D, Sandmaier BM, et al. HLA-matched unrelated donor hematopoietic cell transplantation after nonmyeloablative conditioning for patients with hematologic malignancies. Blood. 2003;102:2021-2030.

28. McSweeney PA, Niederwieser D, Shizuru JA, et al. Hematopoietic cell transplantation in older patients with hematologic malignancies: replacing high-dose cytotoxic therapy with graft- versus-tumor effects. Blood. 2001;97:33903400 .

29. Deeg HJ, Storer B, Slattery JT, et al. Conditioning with targeted busulfan and cyclophosphamide for hemopoietic stem cell transplantation from related and unrelated donors in patients with myelodysplastic syndrome. Blood. 2002;100:12011207.

30. Clift RA, Buckner CD, Thomas ED, et al. Marrow transplantation for chronic myeloid leukemia: a randomized study comparing cyclophosphamide and total body irradiation with busulfan and cyclophosphamide. Blood. 1994;84:2036-2043.

31. Demirer T, Petersen FB, Appelbaum FR, et al. Allogeneic marrow transplantation following cyclophosphamide and escalating doses of hyperfractionated total body irradiation in patients with advanced lymphoid malignancies: a phase I/II trial. Int J Radiat Oncol Biol Phys. 1995;32:11031109.

32. Storb R, Deeg HJ, Whitehead J, et al. Methotrexate and cyclosporine compared with cyclosporine alone for prophylaxis of acute graft versus host disease after marrow transplantation for leukemia. N Engl J Med. 1986;314:729-735.

33. Sullivan KM, Agura E, Anasetti $\mathrm{C}$, et al. Chronic graft-versus-host disease and other late complications of bone marrow transplantation. Semin Hematol. 1991;28:250-259.

34. Sullivan KM. Graft-versus-host disease. In: Thomas ED, Blume KG, Forman SJ, eds. Hematopoietic Cell Transplantation. Malden, MA: Blackwell Sciences; 1999:515-536.

35. Boeckh M, Bowden RA, Gooley T, Myerson D, Corey L. Successful modification of a pp65 antigenemia-based early treatment strategy for prevention of cytomegalovirus disease in allogeneic marrow transplant recipients [letter]. Blood. 1999; 93:1781-1782.

36. Marr KA, Seidel K, Slavin M, et al. Prolonged fluconazole prophylaxis is associated with persistent protection against candidiasis-related death in allogeneic marrow transplant recipients: longterm follow-up of a randomized, placebocontrolled trial. Blood. 2000;96:2055-2061.

37. Boeckh M, Marr KA. Infection in hematopoietic stem cell transplantation. In: Rubin $\mathrm{RH}$, Young LS, eds. Clinical Approach to Infection in the Compromised Host. New York, NY: Kluwer Academic/Plenum Publishers; 2002:527-571.

38. Boeckh M, Gooley TA, Myerson D, Cunningham T, Schoch G, Bowden RA. Cytomegalovirus pp65 antigenemia-guided early treatment with ganciclovir versus ganciclovir at engraftment after allogeneic marrow transplantation: a randomized double-blind study. Blood. 1996;88:4063-4071.

39. Kanda Y, Mineishi S, Saito T, et al. Long-term low-dose acyclovir against varicella-zoster virus reactivation after allogeneic hematopoietic stem cell transplantation. Bone Marrow Transplant. 2001;28:689-692. 
40. Kalbfleisch JD, Prentice RL. The Statistical Analysis of Failure Time Data. New York, NY: John Wiley \& Sons; 1980

41. Harrell FE Jr, Lee KL, Califf RM, Pryor DB, Rosati RA. Regression modelling strategies for improved prognostic prediction. Stat Med. 1984;3: 143-152.

42. Yancik R, Ganz PA, Varricchio CG, Conley B. Perspectives on comorbidity and cancer in older patients: approaches to expand the knowledge base. J Clin Oncol. 2001;19:1147-1151.

43. Piccirillo JF, Tierney RM, Costas I, Grove L, Spitznagel EL Jr. Prognostic importance of comorbidity in a hospital-based cancer registry. JAMA. 2004;291:2441-2447.

44. Kaplan MH, Feinstein AR. The importance of classifying initial co-morbidity in evaluating the outcome of diabetes mellitus. J Chronic Dis. 1974;27:387-404

45. Romano PS, Roos LL, Jollis JG. Adapting a clinical comorbidity index for use with ICD-9-CM administrative data: differing perspectives. J Clin Epidemiol. 1993;46:1075-1079.

46. Cahn J-Y, Labopin M, Schattenberg A, et al. Allogeneic bone marrow transplantation for acute leu- kemia in patients over the age of 40 years. Leukemia. 1997;11:416-419.

47. Gómez-Núñez M, Martino R, Caballero MD, et al Elderly age and prior autologous transplantation have a deleterious effect on survival following allogeneic peripheral blood stem cell transplantation with reduced-intensity conditioning: results from the Spanish multicenter prospective trial. Bone Marrow Transplant. 2004;33:477-482.

48. Molina AJ, Storb RF. Hematopoietic stem cell transplantation in older adults. In: Rowe JM, Lazarus HM, Carella AM, eds. Handbook of Bone Marrow Transplantation. London, United Kingdom: Martin Dunitz; 2000:111-137.

49. Sutton L, Chastang C, Ribaud P, et al. Factors influencing outcome in de novo myelodysplastic syndromes treated by allogeneic bone marrow transplantation: a long-term study of 71 patients Societe Francaise de Greffe de Moelle. Blood. 1996;88:358-365.

50. Hogan WJ, Maris M, Storer B, et al. Hepatic injury after nonmyeloablative conditioning followed by allogeneic hematopoietic cell transplantation: a study of 193 patients. Blood. 2004;103:78-84.

51. Fujimaki K, Maruta A, Yoshida M, et al. Severe cardiac toxicity in hematological stem cell trans- plantation: predictive value of reduced left ventricular ejection fraction. Bone Marrow Transplant. 2001;27:307-310.

52. Loberiza FRJ, Rizzo JD, Bredeson CN, et al. Association of depressive syndrome and early deaths among patients after stem-cell transplantation for malignant diseases. J Clin Oncol. 2002; 20:2118-2126.

53. Gratwohl A, Hermans J, Goldman JM, et al. Risk assessment for patients with chronic myeloid leukaemia before allogeneic blood or marrow transplantation. Lancet. 1998;352:1087-1092.

54. Greenberg P, Cox C, LeBeau MM, et al. International scoring system for evaluating prognosis in myelodysplastic syndromes [erratum appears in Blood. 1998;91:1100]. Blood. 1997;89:20792088.

55. Newschaffer CJ, Bush TL, Penberthy LT. Comorbidity measurement in elderly female breast cancer patients with administrative and medical records data. J Clin Epidemiol. 1997;50:725-733.

56. Katz JN, Chang LC, Sangha O, Fossel AH, Bates DW. Can comorbidity be measured by questionnaire rather than medical record review? Med Care. 1996;34:73-84. 